\title{
UMA INVESTIGAÇÃO SISTEMÁTICA SOBRE O USO DE JOGOS DIGITAIS PARA REABILITAÇÃO
}

\author{
A SYSTEMATIC INVESTIGATION INTO THE USE OF DIGITAL GAMES \\ FOR REHABILITATION
}

\author{
Flávia Gonçalves Fernandes
}

\begin{abstract}
Introduction: Digital games are no longer seen as a form of entertainment detrimental to health. They have become an important tool to improve the treatment of patients, ranging from those who are experiencing a serious illness, such as cancer, for example, to those requiring milder procedures such as physiotherapy. However, there are people who are physically disabled and have difficulty using digital games. Objectives: In this line of reasoning, this work presents a systematic review on the use of digital games for people with physical disabilities in the upper limbs. Methods: The sources of research were the following indexed databases: Portal of Periodicals of the Coordination of Improvement of Personnel of Higher Level (CAPES), Scopus, Science Direct, IEEE Xplore and Scielo. Results: It should be noted that the application of Systematic Mapping in the elaboration of a bibliographical review allowed to identify the main gaps for the development of new research, in addition, it directs to the main publications linked to the study. Conclusion: Finally, the results show that it is an area that is constantly expanding.
\end{abstract}

Universidade Federal de Goiás - UFG

\section{Resumo}

Key words: Disabled persons; Upper limbs; review.
Resumo - Introdução: Os jogos digitais deixaram de ser vistos como uma forma de entretenimento prejudicial à saúde. Eles tornaram-se uma ferramenta importante para melhorar $o$ tratamento dos pacientes, que vão desde aqueles que estão atravessando uma grave enfermidade, como por exemplo o câncer, até os que demandam procedimentos como a fisioterapia. Contudo, há pessoas com deficiência física que possuem dificuldade em utilizar jogos digitais. Objetivos: Nesta linha de raciocínio, este trabalho apresenta uma revisão sistemática sobre o uso de jogos digitais para pessoas com deficiência física nos membros superiores. Métodos: As fontes de pesquisa foram as seguintes bases de dados indexadas: Portal de Periódicos da Coordenação de aperfeiçoamento de Pessoal de Nível Superior (CAPES), Scopus, Science Direct, IEEE Xplore e Scielo. Resultados: Cabe destacar que a aplicação do mapeamento sistemático na elaboração de revisão bibliográfica permitiu identificar as principais lacunas para o desenvolvimento de novas pesquisas, além de direcionar as principais publicações ligadas ao estudo. Conclusão: Por fim, os resultados obtidos mostram que é uma área em constante expansão atualmente.

Palavras chave: Pessoas com deficiência; Membros superiores; Revisão sistemática. 


\section{Introdução}

Muitas vezes, pessoas com deficiência física ou mental são vítimas de preconceito e discriminação. Também, há o costume de não receber o mesmo tipo de tratamento e ter a liberdade de ir e vir prejudicada pelas más condições de vias de acesso público e privado.

Porém, a Declaração Universal dos Direitos Humanos deixa claro que todas as pessoas devem ser tratadas fraternalmente, independente de deficiências. No caso específico do Brasil, a Constituição Federal define como meta a busca do bem-estar de todos, sem quaisquer tipos de discriminação. Da mesma maneira, o Código Penal brasileiro determina como passível de punição os atos criminosos e de desrespeito causados por fatores discriminatórios ${ }^{1}$.

Pode-se definir a deficiência física como diferentes condições motoras que acometem as pessoas comprometendo a mobilidade, a coordenação motora geral e da fala, em consequência de lesões neurológicas, neuromusculares, ortopédicas, ou malformações congênitas ou adquiridas².

Segundo a Declaração de Salamanca, as diferenças humanas são normais e a aprendizagem deve se adaptar às necessidades das crianças ao invés de se adaptar a criança a assunções preconcebidas a respeito do ritmo e da natureza do processo de aprendizagem ${ }^{1}$.

Além disso, o longo tempo necessário para a promoção da consciência corporal, aceitação da deficiência e a pouca motivação gerada pelos métodos tradicionais são apontados como motivo de abandono do tratamento, caracterizando-se como uma das principais causas de falha terapêutica ${ }^{3}$. Outro problema encontrado por pessoas com deficiência física é a falta de recursos que possibilitem dar continuidade no tratamento domiciliar, com pouco ou nenhum monitoramento presencial de um terapeuta ${ }^{4}$.

Ainda é importante lembrar que, geralmente, as pessoas que nascem com malformação congênita de algum membro do corpo humano, também possuem outros problemas de saúde, que podem ser físicos (em outros membros), mentais, cardiovasculares, respiratórios, entre outros.

Assim, a utilização do jogo digital pode tornar-se uma alternativa em proporcionar maior motivação nas brincadeiras por meio de desafios com técnicas virtuais, trabalhando conceitos que podem auxiliar na cognição, nos aspectos emocionais e físicos dos pacientes, no favorecimento dos movimentos do membro afetado, lazer, socialização e convivência com outras pessoas.

Nessa perspectiva, a promoção da consciência corporal de forma virtual por meio de jogos visa simular situações reais; também se percebe que o uso dela melhora a funcionalidade dos membros acometidos e o leva a retomar as atividades nas áreas de desempenho ocupacional ${ }^{5}$.

Portanto, é importante a realização de pesquisas cujo o objetivo é fazer com que pessoas com deficiência física também possam ter ao seu alcance os benefícios da utilização dos jogos digitais.

Neste contexto, o objetivo deste trabalho é verificar o panorama das pesquisas relacionadas sobre a aplicação de jogos digitais para pessoas com deficiência física nos membros superiores, apresentando um estudo sistêmico acerca do que vem sendo publicado para tal vertente.

\section{Metodologia}

Para a revisão da literatura, foi desenvolvido um mapeamento sistemático (mapping study), de acordo com a metodologia proposta por 6 e 7, que consiste na busca de estudos cadastrados em bases de dados por meio de operadores lógicos para a seleção dos artigos, a partir da seleção de palavras ou expressões chave.

Esta técnica de mapeamento sistemático também foi utilizada por ${ }^{8,9} \mathrm{e}^{10}$. As bases de dados consideradas foram: Portal de Periódicos da Coordenação de Aperfeiçoamento de Pessoal de Nível Superior - CAPES 11, Scopus 12, Science Direct 13, IEEE Xplore 14 e Scielo 15, bases disponíveis na Universidade Federal de Goiás. Vale ressaltar que foram analisados somente artigos de periódicos revisados por pares.

As expressões lógicas empregadas foram: "games" AND "disability" AND "upper limbs" AND "wearable". Foram escolhidas estas strings para buscar trabalhos que envolvam jogos digitais para pessoas com deficiência física nos membros superiores e que utilizam dispositivos vestíveis para adaptação, reabilitação ou inserção deste público no contexto social.

Após a busca inicial, foram adotados como filtros: o idioma (inglês e português); sem restrição da área de conhecimento e o tipo de publicação (artigo de periódico revisado por pares). Aplicados os filtros em cada base de dados, os artigos foram classificados pelo ano de publicação e pelos títulos, observando as possíveis repetições entre as bases de dados. 
A etapa final de seleção dos artigos foi efetuada a partir da leitura e análise dos títulos e resumos, de modo a excluir aqueles trabalhos que não se relacionavam diretamente ao tema em estudo, sendo a revisão da bibliografia desenvolvida a partir deste resultado obtido.

Para isso, os critérios utilizados para leitura dos títulos e resumos dos artigos foram: se continham as palavras-chaves, e se as mesmas estavam aderentes ao contexto almejado que, neste caso, refere-se às pesquisas envolvendo jogos digitais para pessoas com deficiência física nos membros superiores e/ou utilizando dispositivos vestíveis. Neste sentido, trabalhos que abordavam temáticas diferentes foram descartados.

Após a realização das buscas nas bases de dados, os resultados obtidos foram organizados em forma de gráficos e tabelas com a finalidade de apresentá-los de maneira mais prática. A Tabela 1 apresenta os resultados totais obtidos no mapeamento e a Figura 1 apresenta a evolução da publicação dos artigos de periódicos levantados, considerando-se as citadas palavraschave e a data limite de junho de 2018.

Tabela 1. Síntese dos resultados obtidos nas pesquisas nas bases de dados.

\begin{tabular}{|l|c|c|}
\hline & \multicolumn{2}{|c|}{$\begin{array}{c}\text { Expressões-chave: } \\
\text { "Games" AND "disability" } \\
\text { AND "upper limbs" AND } \\
\text { "wearable" }\end{array}$} \\
\hline \multicolumn{1}{|c|}{ BASES DE DADOS } & \multicolumn{2}{|c|}{ Número de artigos } \\
\hline Periódicos Capes & Inicial & Final \\
\hline Scopus & 509 & 6 \\
\hline Science Direct & 107 & 4 \\
\hline IEEE Xplore & 187 & 10 \\
\hline Scielo & 67 & 7 \\
\hline Total & 20 & 2 \\
\hline & 823 & 29 \\
\hline
\end{tabular}

Algumas bases de dados apresentaram grande número de artigos repetidos entre si, e vinte artigos dessas bases que foram classificados em aderentes quanto ao título e resumo possuíam acesso restrito ao texto completo, inviabilizando a sua utilização.

Dessa forma, na seleção final, por meio do mapeamento sistemático foi criado um banco de dados com 29 (vinte e nove) referências de bases de dados para a string "Games" AND "disability" AND "upper limbs" AND "wearable".

A evolução da publicação anual dos artigos selecionados na busca internacional pode ser vista na Figura 1, onde é possível notar que os artigos envolvendo o assunto abordado neste trabalho foram publicados nas últimas décadas.

Dessa forma, observa-se que o assunto é recente e apresenta a tendência de expansão na pesquisa. Uma possível explicação para este fato é uma maior maturidade na aplicação de jogos nas mais diversas áreas e na conscientização da necessidade de inclusão de pessoas com deficiência física.

Os periódicos em que foram publicados artigos sobre estes tópicos são apresentados na Tabela 2.

Assim, percebe-se que os principais periódicos que publicaram sobre o tema recentemente foram "Disability and Rehabilitation: Assistive Technology" e "Revista Brasileira de Engenharia Biomédica". Ambas são revistas internacionais e multidisciplinares.

A primeira tem como missão promover e compartilhar a ciência interdisciplinar e integradora de serviços de tecnologia assistiva para as pessoas com deficiência, doenças crônicas e desafios para o desempenho das atividades e a participação nos papéis vitais, alcançar um melhor funcionamento e qualidade de vida.

Já a segunda tem como objetivo fornecer material didático e atualização profissional, além de servir como um fórum para o estabelecimento de políticas de desenvolvimento e incorporação de tecnologias de saúde pelos setores público ou privado.

Esta revista multidisciplinar visa leitores e autores com interesse em usar ou desenvolver ferramentas baseadas em engenharia e ciências físicas para compreender e resolver problemas nas ciências biológicas e médicas. Entretanto, observa-se que não há uma grande discrepância na quantidade de publicações entre os periódicos. Isto pode ser explicado em parte pelo 
baixo número de trabalhos selecionados nesta pesquisa.

Figura 1. Evolução da publicação de artigos levantados em função das palavras-chave empregadas no mapeamento. Número de Publicações por Período

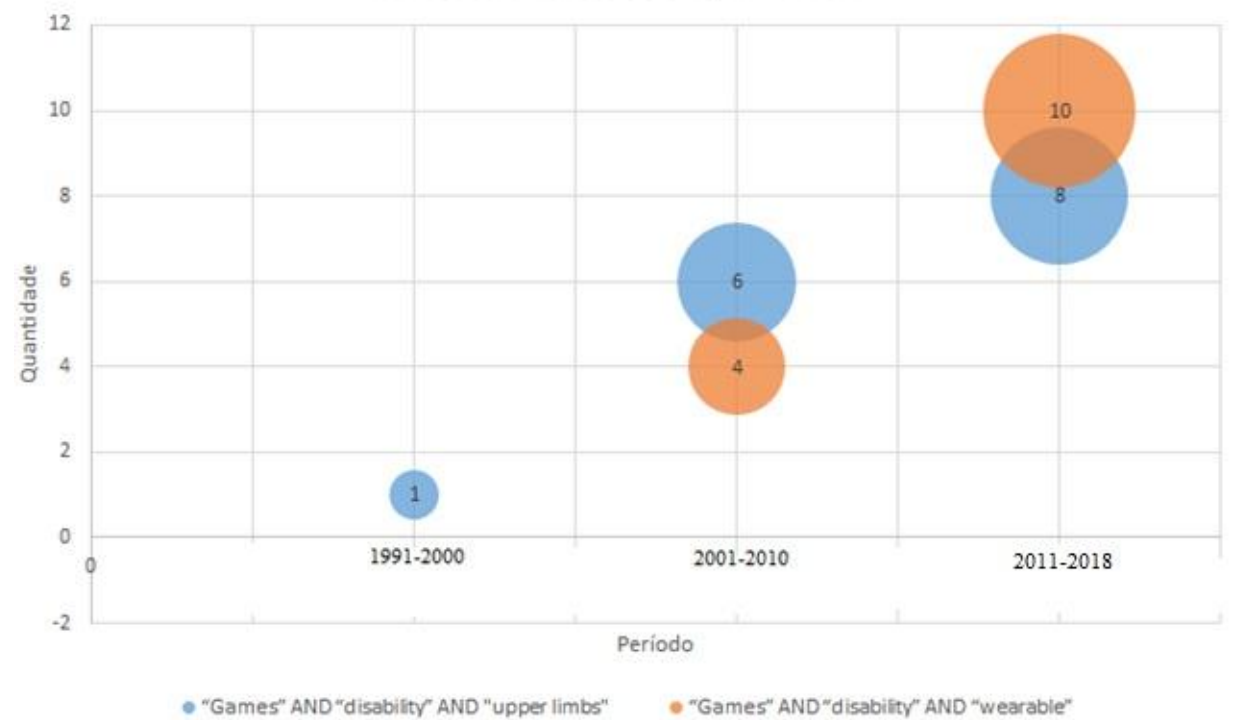

Tabela 2. Principais periódicos sobre "jogos digitais para pessoas com deficiência física nos membros superiores e/ou utilizando dispositivos vestíveis".

\begin{tabular}{|c|c|}
\hline Periódico & Número de artigos publicados \\
\hline Disability and Rehabilitation: Assistive Technology & 4 \\
\hline Revista Brasileira de Engenharia Biomédica & 4 \\
\hline Internacional Joumal of Developmental Disabilities & 3 \\
\hline Biosystems and Biorobotics & 2 \\
\hline Journal of Pediatric Rehabilitation Medicine & 2 \\
\hline Boumal on Applied System Innovation for Modern Technology & 2 \\
\hline Medical Joumal of Malasya & 2 \\
\hline Bnternational Joumal of Social Robotics & 2 \\
\hline Trials & 2 \\
\hline International Joumal on Technology and Innovation in Health & 2 \\
\hline International Joumal on Modelling and Simulation & 2 \\
\hline International Joumal Gamification for Human Integration & 1 \\
\hline
\end{tabular}

A Figura 2 apresenta um gráfico de barras de forma horizontal com o número de artigos de periódicos publicados por países. Os trabalhos publicados são de diferentes países, tais como: Estados Unidos, Alemanha, Brasil, China, Áustria, Itália, França, Japão e Coréia do Sul. Na América do Sul, foram encontrados trabalhos desenvolvidos apenas no Brasil que abordem esse tema.

Em seguida, os 29 artigos foram lidos de forma integral. E foi descoberto que vários deles não estavam de fato relacionados com o tema em questão, mesmo após a seleção pela leitura dos títulos e resumos dos artigos. Os artigos descartados abordavam conceituações teóricas sobre jogos digitais, pessoas com deficiência física e/ou dispositivos vestíveis, nem sempre relacionados entre si. Porém, os temas não tinham abrangência na área da tecnologia aplicada, ou seja, apresentavam ideias e temáticas sobre determinado assunto, contudo, não apresentavam aplicações com resultados.

Além disso, outros trabalhos tratavam-se de revisões sistemáticas, como 16 e 17, por exemplo. Contudo, o presente trabalho se diferencia das outras revisões por focar em membros superiores e dispositivos vestíveis (wearables), que é algo mais prático, fácil de utilizar e inovador, enquanto as demais abordaram ferramentas tecnológicas diversas e também variados membros do corpo humano.

A partir disso, restaram apenas onze artigos 
relacionados ao tema em questão, os quais serão detalhados na próxima seção do artigo.

Apesar de apresentarem escopos diferentes, os ratificação da relevância do uso de sistemas de interação natural para promoção da consciência corporal dos membros superiores ou interações alternativas.

Figura 2. Países referentes aos trabalhos selecionados.

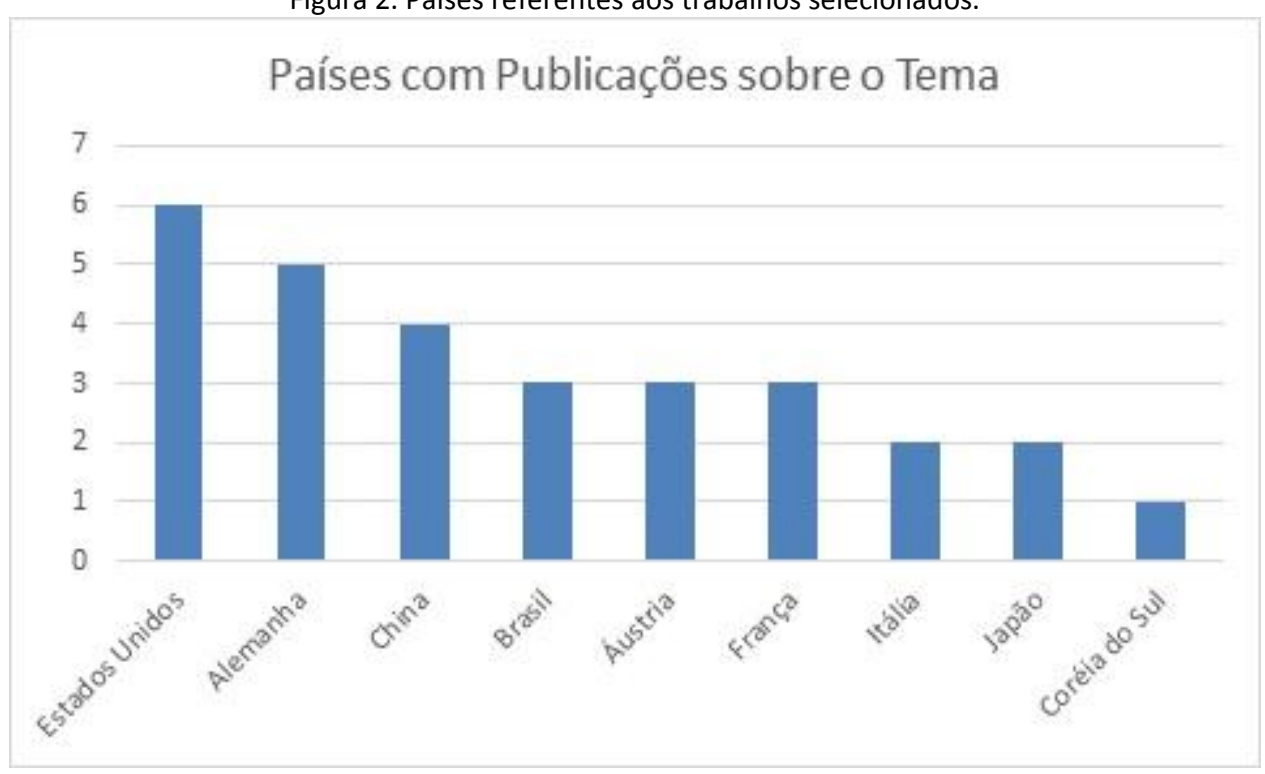

Os trabalhos selecionados pela investigação sistemática são apresentados de forma mais detalhada a seguir.

\section{A. Trabalho T1}

O Trabalho T1, denominado "Game Console Controller Interface for People with Disability", apresenta um dispositivo de interface que permite às pessoas com deficiência nos membros superiores brincarem com videogames de console. Este protótipo é um dispositivo completamente novo conectado entre o controlador original e o console. Permite a conexão da interface mais adequada para a deficiência específica. Graças a este dispositivo, a interface específica atua da mesma forma que os botões de pressão e/ou joysticks do controlador original. O mapeamento de funções de sensores externos para o controlador original é realizado por um procedimento simples e intuitive ${ }^{18}$.

B.

Trabalho T2

Os autores deste trabalho realizaram um estudo, que consiste em verificar a contribuição de um programa de jogos e brincadeiras adaptados para o desenvolvimento de alunos com deficiência física, realizado em uma escola estadual que possui salas especiais para deficientes físicos. Primeiramente, foi feita avaliação das habilidades e dificuldades dos alunos; a seguir foi elaborado e aplicado um programa de intervenção. O programa contou com dezessete atividades adaptadas que foram desenvolvidas em vinte e duas aulas, com duração de uma hora cada. A partir dos resultados obtidos, concluiu-se que a proposta contribuiu de maneira positiva para o desenvolvimento físico, acadêmico, emocional e social de alunos com deficiência física no ambiente escolar ${ }^{19}$.

\section{Trabalho T3}

O Trabalho T3 é intitulado “A reabilitação de pessoas com deficiência através do desporto adaptado". O objetivo deste estudo foi realizar uma revisão de literatura sobre a reabilitação de pessoas com deficiência através do desporto adaptado, baseada em referências nacionais e internacionais obtidas através da base de dados da CAPES, Pubmed, Scielo e na base de dados da biblioteca da Faculdade de Desporto da Universidade do Porto - Portugal. Observou-se que os benefícios da prática desportiva são evidenciados por grande parte de estudiosos da área do desporto adaptado e contribuem para a qualidade de vida desta população ${ }^{20}$.

\section{Trabalho T4}

O Trabalho T4 retrata a inclusão escolar do 
aluno com deficiência física: contribuições da terapia ocupacional. O objetivo deste estudo foi o de identificar as dificuldades de uma professora referentes ao processo de inclusão de um aluno com deficiência física, adaptar recursos pedagógicos, adequar mobiliário escolar e orientar a professora em situações específicas. A análise de dados evidenciou que, com base na análise e na adequação da interação entre as demandas funcionais da pessoa, da tarefa e do ambiente, é possível o aluno responder satisfatoriamente aos desafios inerentes ao contexto educacional, e destacaram a importância da parceria entre o professor e o terapeuta ocupacional nesse processo ${ }^{21}$.

\section{E. Trabalho T5}

O Trabalho T5, "Virtual Reality as Adjunctive Therapy for Upper Limb Rehabilitation in Cerebral Paralisys", apresenta um jogo que utiliza Realidade Virtual como terapia adjuvante para a reabilitação dos membros superiores de crianças vítimas de paralisia cerebral. Foi avaliado o nível de cooperação e satisfação das crianças ao praticar movimentos de braço e mão durante atividades lúdicas em um ambiente físico, em comparação com um ambiente virtual baseado em captura de vídeo usando cinco graus de liberdade. Embora a cooperação tenha sido semelhante em ambos os ambientes, as crianças expressaram maior satisfação com o ambiente físico do que com o ambiente virtual. As razões para isso podem incluir dificuldade ou frustração com as atividades virtuais e/ou adequação dos jogos de computador ${ }^{22}$.

\section{F. Trabalho T6}

O Trabalho T6, "Upper Extremity Rehabilitation of Children with Cerebral Palsy using Accelerometer Feedback on a Multitouch Display", apresenta um sistema para reabilitação para membros superiores de crianças com paralisia cerebral usando feedback de acelerômetro em um visor multitoque. O sistema permite que as crianças se envolvam em cenários de jogos interativos, enquanto realizam intensamente os exercícios desejados. Para incentivar a postura correta durante os exercícios terapêuticos, foi usado um sensor cinemático sem fio, usado no tronco do paciente, como um canal de feedback para os jogos. O sistema passou por várias fases de projeto, incorporando a entrada de observações de terapia e sessões clínicas, bem como feedback de profissionais médicos ${ }^{23}$.

\section{G. Trabalho T7}

O Trabalho T7 "Development of an EMGACC-Based Upper Limb Rehabilitation Training System" tem como tema principal o desenvolvimento de um sistema de treinamento de reabilitação de membros superiores projetado para uso de crianças com paralisia cerebral. Os autores procuraram satisfazer os requisitos de treinamento em casa, aproveitando a combinação de sensores de acelerômetros portáteis e eletromiografia de superfície usados no membro superior para capturar movimentos funcionais. Os resultados de um treinamento de longo prazo realizado com três sujeitos de paralisia cerebral demonstraram que eles poderiam melhorar o desempenho do jogo por meio de treinamento repetitivo, sendo necessário treinamento persistente para melhorar o efeito de reabilitação ${ }^{24}$.

\section{H. Trabalho T8}

O Trabalho T8, "Pediatric rehabilitation with the reachman's modular handle", apresenta os resultados de um estudo preliminar com uma criança com paralisia cerebral utilizando o ReHaptic Handle, um novo dispositivo robótico para a reabilitação pediátrica da função do membro superior.

Jogos de computador interativos foram implementados para aumentar a participação dos participantes e engajamento, promovendo, assim, a recuperação motora.

O pinçamento, com o indicador e o polegar, a supinação/pronação do antebraço, bem como a flexão/extensão do punho, foram treinados duas ou três vezes por semana durante quinze minutos cada. Observou-se um aumento da precisão e lisura do movimento de supinação/pronação do antebraço com o indivíduo, bem como uma redução na duração do movimento 25 .

\section{Trabalho T9}

O Trabalho T9, "Hand Therapist: a rehabilitation approach based on wearable technology and video gaming", trata-se de um sistema de reabilitação da mão, principalmente para pacientes vítimas de Acidente Vascular Cerebral (AVC), composto por: braçadeira Myo, luva robótica e a game engine Unity 3D. Esta abordagem apresenta uma solução que combina desempenho, baixo custo e motivação para terapia da mão ${ }^{26}$. 
Trabalho T10

O Trabalho T10, "Hand Posture and Gesture Recognition using Myo Armband and Spectral Collaborative Representation based Classification", propõe o uso da representação colaborativa baseada em Spectral Domain para reconhecer as posturas e gestos das gravações eletromiografia (EMG) adquiridos por um sensor recentemente introduzido: braçadeira Myo Thalmic Labs.

A precisão de reconhecimento obtida para um conjunto de seis gestos e posturas é promissor, com uma precisão superior a 97\%, o que é um resultado eficiente na literatura relacionada. Os algoritmos são desenvolvidos para a criação de uma interface homem-máquina intuitiva para navegar em uma cadeira de rodas robótica ${ }^{27}$.

\section{K. Trabalho T11}

O Trabalho T11 trata-se de uma estratégia para suportar interação humano-computador de crianças com deficiência nos membros superiores por meio do dispositivo vestível Myo, com o objetivo de ampliar o uso de jogos digitais para o público-alvo. Dessa forma, foi realizada a adaptação de jogos digitais para crianças que possuem deficiência física nos membros superiores, seja por malformação congênita e/ou adquirida, com a finalidade de que seja possível a utilização dos jogos digitais por este público e também auxiliar na aceitação da deficiência.

Para o seu desenvolvimento, foram utilizadas as funcionalidades do dispositivo vestível Myo para controlar um jogo de quebracabeça como meio de prover a interação entre o indivíduo e o jogo, visto que o Myo é um bracelete que oferece facilidade e praticidade de uso. Assim, procurou-se oferecer a estas crianças uma ferramenta adicional (os jogos digitais) para o processo de promoção da consciência corporal e ampliar sua acessibilidade a jogos ${ }^{28}$.

Neste contexto, o jogo é controlado pelos movimentos do membro superior do usuário, que apresenta a deficiência, por meio do Myo.

Assim, a longo prazo, pode-se auxiliar na aceitação da limitação motora, motivar os pacientes a utilizar com maior frequência o membro com deficiência, desenvolver e criar habilidades, despertar potenciais, conhecer novas tecnologias, melhorar os aspectos cognitivos, emocionais e físicos, a socialização e o lazer.

Os trabalhos citados confirmaram que a utilização dos jogos digitais ajuda a estimular os usuários a realizarem atividades do mundo real. Além disso, sabe-se que o uso de interfaces naturais é recente e estimulante, uma vez que são elementos naturais humanos, quase imperceptíveis quando o usuário está imerso na aplicação. Por isso, as Interfaces Naturais de Usuário (NUI) são utilizadas em vários trabalhos analisados.

Adaptação é importante para ampliar as potencialidades cognitivas das Pessoas com Necessidades Educacionais Especiais (PNEEs), o que é um dos grandes desafios do trabalho de inclusão no contexto escolar e social. Mas, mesmo com poucos recursos, é possível oferecer boas alternativas para atender às peculiaridades das pessoas com deficiência física adaptando materiais e equipamentos do cotidiano. Estas adaptações permitem que as pessoas com deficiência sejam capazes de se expressar, elaborar perguntas, resolver problemas e se tornar mais participativos, permitindo assim uma maior interação social com outras pessoas.

A relevância é ressaltada em ambientes relacionados com a área médica que necessitam de um processo de reprodução ou repetição de ações e/ou movimentos que produzem um cenário motivacional maior do que a metodologia tradicional.

O trabalho T1, apresentado na seção IV. Trabalhos Selecionados, apresenta um controlador adaptado para pessoas com deficiência física nos membros superiores. Porém, o trabalho T11 utiliza um dispositivo vestível para adaptação controlado por gestos do próprio usuário, o que é algo mais prático e fácil de usar, além de ser uma tecnologia inovadora e atrativa para as pessoas.

Controladores de console típicos são equipados com vários botões e joysticks muitas vezes para ser operado ao mesmo tempo, tornando-se uma barreira real. Algumas soluções do-it-yourself existem adaptando controladores comerciais para necessidades especiais. Mas, desta forma, o controle do videogame é totalmente deixado para os deficientes e muitas vezes ele ainda não é capaz de jogar com suas funcionalidades residuais. Além disso, tais soluções não são nem comercializáveis, sendo modificações não certificadas de um dispositivo comercial.

Os trabalhos T2, T3 e T4 mostram adaptações de jogos em atividades físicas escolares para pessoas com deficiência física, as quais são importantes para permitir a acessibilidade, a independência e a inclusão de crianças e adolescentes no contexto escolar, além de auxiliar na redução do 
preconceito e da discriminação que estas pessoas costumam sofrer nas escolas e na sociedade.

Nos trabalhos T5, T6, T7 e T8, podem ser vistas aplicações para crianças que tiveram paralisia cerebral, com a finalidade de auxiliá-las a retornar os movimentos de seus membros superiores normalmente. Estes protótipos são importantes, visto que a paralisia cerebral é uma doença neurológica não progressiva, causada por distúrbios do cérebro em desenvolvimento.

A terapia física e ocupacional, se iniciada em tenra idade, pode ajudar a minimizar complicações, como contraturas conjuntas, e pode melhorar a amplitude de movimentos e a coordenação dos membros. Embora as formas atuais de terapia para crianças com paralisia cerebral sejam eficazes na minimização dos sintomas, muitas crianças acham que são chatas ou repetitivas.

Os trabalhos T9 e T10 apresentam pesquisas realizadas utilizando o dispositivo vestível Myo. Em T9, a aplicação é uma forma de terapia de mão voltada para pacientes vítimas de AVC, que não possuem deficiência física, mas dificuldade de manuseio de objetos.

Nesse sistema, o usuário faz diversos exercícios repetitivos para recuperar os movimentos da mão e, além do Myo, utilizam uma luva com sensores. Em T10, o protótipo foi criado para pessoas que utilizam cadeira de rodas.

Assim, o usuário movimenta a cadeira de rodas por meio de seus próprios gestos do braço em que está colocado o Myo. Neste caso, a deficiência das pessoas dá-se nos membros inferiores.

Nestes dois projetos, o meio de interação poderia ser trocado para Kinect e joystick, respectivamente, o que provavelmente atingiria o mesmo resultado. Porém, no trabalho T11, estas outras ferramentas tecnológicas não poderiam ser utilizadas, uma vez que as pessoas possuem deficiência física nos membros superiores e têm dificuldade em utilizar as ferramentas citadas.

A Tabela 3 apresenta os tópicos abordados em cada trabalho, comparando-os entre si. Os trabalhos são especificados com siglas (T1 a T11), obedecendo a ordem de apresentação dos mesmos no texto.

As informações presentes na Tabela 3 propiciam concluir que, dentre os trabalhos selecionados, todos abordam adaptação de jogos para membros superiores. O material utilizado para adaptação usado para cada trabalho foi escolhido em razão da aderência ao problema a ser solucionado e do amparo informacional.

Tabela 3. Comparação entre os trabalhos selecionados.

\begin{tabular}{|c|c|c|c|c|}
\hline \multicolumn{1}{|c|}{ Categoria } & Trabalhos & $\begin{array}{c}\text { Membros } \\
\text { Superiores }\end{array}$ & $\begin{array}{c}\text { Membros } \\
\text { Inferiores }\end{array}$ & $\begin{array}{c}\text { Métodos } \\
\text { Utilizados }\end{array}$ \\
\hline Adaptação de jogos digitais & $\mathrm{T} 1$ & Sim & Não & Joystick \\
\hline \multirow{3}{*}{ Adaptação de jogos para inclusão escolar } & $\mathrm{T} 2$ & Sim & Sim & Brincadeiras \\
\cline { 2 - 5 } & $\mathrm{T} 3$ & Sim & Sim & Desporto \\
\cline { 2 - 5 } & $\mathrm{T} 4$ & Sim & Sim & Recursos pedagógicos \\
\hline \multirow{2}{*}{$\begin{array}{c}\text { Jogo para reabilitação de paralisia } \\
\text { cerebral }\end{array}$} & $\mathrm{T} 5$ & Sim & Não & Realidade Virtual \\
\cline { 2 - 5 } & $\mathrm{T} 6$ & Sim & Não & Plataforma Android \\
\cline { 2 - 5 } & $\mathrm{T}$ & Sim & Não & Display multitoque \\
\cline { 2 - 5 } & $\mathrm{T} 8$ & Sim & Não & Robótica \\
\hline Jogo para reabilitação de AVC & $\mathrm{T} 9$ & Sim & Não & Myo \\
\hline Jogo para reabilitação de cadeirantes & $\mathrm{T} 10$ & Não & Sim & Myo \\
\hline Ampliação do uso de jogos digitais & T11 & Sim & Não & Myo \\
\hline
\end{tabular}

A área de aplicação da adaptação, na grande maioria dos trabalhos, está relacionada a área escolar, principalmente aprendizagem e inclusão social, demonstrando a importância do uso de procedimentos alternativos aos tradicionais para os mais variados tipos de tratamento. $E$ os outros trabalhos envolvem criação de jogos para reabilitação de pessoas com deficiência física ou limitação motora devido à paralisia cerebral ou AVC.

Outro aspecto presente no trabalho T11, que o diferencia dos demais é a característica motivacional, em que o jogo se beneficia dos recursos digitais para promover maior imersão e interatividade das pessoas e, consequentemente, maior motivação dos usuários para utilizar a aplicação, o que ocasiona resultados satisfatórios no processo.

\section{Conclusões}

Por meio do mapeamento realizado, foi possível verificar que houve um crescimento no estudo da aplicação de jogos digitais para pessoas com deficiência física nos membros superiores, visto que é uma forma de 
entretenimento e lazer.

Assim, cabe destacar que a aplicação do mapeamento sistemático na elaboração de revisão bibliográfica permite identificar as principais lacunas para o desenvolvimento de novas pesquisas. Além disso, direciona para as principais publicações ligadas ao estudo.

Em virtude do que foi apresentado neste trabalho, verifica-se que há um crescente interesse em pesquisar e publicar nesta área relacionada a jogos digitais para pessoas com deficiência física nos membros superiores e/ou utilizando dispositivos vestíveis.

Inicialmente, a principal linha de pesquisa nesta área é voltada para reabilitação de pessoas com deficiência física ou que estão com paralisia cerebral, pois sofreram acidente vascular cerebral. Porém, as tendências atuais são inserir cada vez mais a tecnologia no cotidiano das pessoas. Logo, os métodos de pesquisa mais trabalhados são voltados para jogos digitais interativos para as pessoas com deficiência com a finalidade de auxiliar no processo de inclusão social, escolar e digital.

Com isso, percebe-se que a utilização de interface natural (NUI) é uma tendência para auxiliar no processo de inclusão de pessoas com deficiência, como a realidade virtual e o dispositivo vestível Myo, por exemplo, visto que são técnicas novas e mais atrativas, já que exploram os movimentos do próprio usuário, em tempo real. Nota-se, portanto, que há espaço para pesquisas de novos dispositivos capazes de oferecer uma melhor interação das pessoas com deficiência com os jogos digitais.

Ainda é importante ressaltar que os dispositivos vestíveis são tecnologias assistivas inovadoras e podem auxiliar no processo de reabilitação de pacientes, fisioterapia, aceitação da deficiência, lazer, entretenimento, adaptação e inclusão social.

Assim, um recurso ainda pouco utilizado identificado a partir da pesquisa efetuada são voltadas para a utilização de dispositivos vestíveis em jogos digitais para pessoas com deficiência física e que poderia ter bons resultados nesta área, devido à sua praticidade e facilidade de uso.

Além disso, apesar de ser verificado um aumento na quantidade de trabalhos publicados abordando o tema desta pesquisa, observa-se que é um número pequeno em face a quantidade de pessoas e situações distintas que necessitam se beneficiar da utilização dos jogos digitais.

Outra carência observada é a falta de métricas e/ou protocolos para medir os ganhos/benefícios obtidos pelas pessoas com deficiência ao utilizarem os jogos digitais. Vale ressaltar que, conforme já mencionado, algumas dessas pessoas possuem deficiências adicionais, como, por exemplo, a mental.

Portanto, percebe-se a necessidade de fomentar esta área de pesquisa para oferecer a este público com deficiência física acesso ao jogo digital como forma de tratamento, aquisição de conhecimento, motivação, entretenimento ou mesmo por inclusão.

Dessa forma, poderá se obter uma maior maturidade nos resultados obtidos e assim promover uma sistematização no emprego dos jogos digitais no auxílio da promoção do bem estar destas pessoas.

\section{Referências}

1. Guia de direitos. Discriminação com Deficientes. [Internet]. 2016. [citado em 20 ago. 2017]. Disponível em: http://www.guiadedireitos.org/index.php?option $=$ com_content $\&$ view $=$ article $\& i d=1040 \&$ Itemid $=2$ 64

2. Kouroupetroglou, G. Disability Informatics and Web Accessibility for Motor Limitations. IGI Global, 2013.

3. Dias, RS; Sampaio, ILA; Taddeo, LS. Fisioterapia: A Introdução do Lúdico no processo de reabilitação de pacientes em tratamento fisioterapêutico. In: VIII Brazilian Symposium on Games and Digital Entertainment. 4. Rio de Janeiro-RJ, 2009.

4. Botella, C et al. Treating cockroach phobia with augmented reality. Behavior Therapy, v.41. n. 3, p. 401-413, 2010.

5. Grande, AAB; Galvão, FRO; Gondim, LCA. Reabilitação virtual através do videogame: relato de caso no tratamento de um paciente com lesão alta dos nervos mediano e ulnar. Revista Acta Fisiátrica, Rio Grande do Norte, v. 18, n. 3, p. 157162, 2011.

6. Bailey, J; Budgen, D; Turner, M; Kitchenham, B; Brereton, P; Linkmon, S. Evidence relating to Object-Oriented software design: $A$ survey. First International Symposium on Empirical Software Engineering and Measurement. Computer Society, 2007.

7. Petersen, K; Feldt; R; Mujtaba, S; Mattsson, M. Systematic Mapping Studies in Software Engineering. School of Engineering, Blekinge Institute of Technology. University of Bari, Italy, 26 - 27 June. 2008.

8. Paula, HMD; Ilha, MSDO. Uso da Moringa oleifera no tratamento de águas residuárias de usinas de concreto: mapeamento 
sistemático. REEC - Revista Eletrônica de Engenharia Civil, v. 11, n. 1, p. 50 - 60, 2016.

9. Bonfim, WB; Baldin, V; Pereira, RR; Paula, HM. Lama residual de usinas de concreto: características e aplicações na confecção de blocos. REEC - Revista Eletrônica de Engenharia Civil, v. 13, n. 2, p. 32 - 43, 2017.

10. Rodrigues, KC; Mesquita, HC; Eduardo, RC; Paula, HM. Mapeamento sistemático de referências do uso do BIM na compatibilização de projetos na construção civil. REEC - Revista Eletrônica de Engenharia Civil, v. 13, n. 1, p. 219 239, 2017.

11. Capes - Portal de Periódicos da Coordenação de Aperfeiçoamento de Pessoal de Nível Superior. [Internet]. 2018. [citado em 18 jun. 2018]. Disponível em: http://wwwperiodicos-capes-gov-

br.ez49.periodicos.capes.gov.br

12. Scopus. [Internet]. 2018. [citado em 18 set. 2018]. Disponível em: https://www.scopus.com

13. Science Direct. [Internet]. 2018. [citado em 18 set. 2018]. Disponível em: http://www.sciencedirect.com

14. IEEE Xplore. [Internet]. 2018. [citado em 18 set. 2018]. Disponível em: https://ieeexplore.ieee.org/

15. Scielo. [Internet]. 2018. [citado em 18 set. 2018]. Disponível em: www.scielo.org/

16. Rego, P; Moreira, PM; Reis, LP. Serious Games for Rehabilitation: A Survey and a Classification Towards a Taxonomy. In: 5th Iberian Conference on Information Systems and Technologies, 2010.

17. Bonnechere, B;Jansen, B;Omelina, L;Van Sint Jan, S. The use of commercial video games in rehabilitation: a systematic review. In: International journal of rehabilitation research, 2016.

18. lacopetti, $F$ et al. Game Console Controller Interface for People with Disability. In: Computer Society IEEE, p. 757-762, 2008.

19. Braccialli, LMP; Manzini, EJ; Reganhan, WG. Contribuição de um programa de jogos e brincadeiras adaptados para a estimulação de habilidades motoras em alunos com deficiência física. In: REUNIÃO ANUAL DA ANPED, 27., 2004. Anais... 2004.

20. Cardoso, VD. A reabilitação de pessoas com deficiência através do desporto adaptado. Revista Brasileira de Ciências no Esporte, Florianópolis, v. 33, n. 2, p. 529-539, abr./jun. 2011.

21. Paula, AFM.; Baleotti, LR. Inclusão escolar do aluno com deficiência física: contribuições da terapia ocupacional. 2011. Cadernos de Terapia Ocupacional da UFSCar, São Carlos, v. 19, n.1, p. 53-69, 2011.

22. Guberek, $\mathrm{R}$ et al. Virtual Reality as Adjunctive Therapy for Upper Limb Rehabilitation in Cerebral Palsy. Virtual Rehabilitation International Conference. 2009.

23. Dunne, A et al. Upper Extremity Rehabilitation of Children with Cerebral Palsy using Accelerometer Feedback on a Multitouch Display. 32nd Annual International Conference of the IEEE EMBS, Buenos Aires-Argentina, 2010.

24. Liu, L et al. Development of an EMGACC-Based Upper Limb Rehabilitation Training System. IEEE Transactions on Neural Systems and Rehabilitation Engineering, 2016.

25. Tong, LZ et al. Pediatric rehabilitation with the reachMAN's modular handle. Procedings IEEE Eng. Med. Biol. Soc. 2015; 2015:3933-6. doi: 10.1109/EMBC.2015.7319254, 2015.

26. Lipovský, R; Ferreira, HA. Hand Therapist: a rehabilitation approach based on wearable technology and video gaming. Portuguese BioEngineering Meeting. 4. Porto: Portugal, 2015.

27. Boyali, A; Hashimoto, N; Matsumoto, $\mathrm{O}$. Hand Posture and Gesture Recognition using Myo Armband and Spectral Collaborative Representation based Classification. In: IEEE 4th Global Conference on Consumer Electronics GCCE, 2015.

28. Fernandes, FG. Uma estratégia para suportar interação humano-computador de crianças com deficiência nos membros superiores por meio de dispositivo vestível. 2017. 152 p. Dissertação (Mestrado) - Programa de PósGraduação em Engenharia Biomédica da Faculdade de Engenharia Elétrica, Universidade Federal de Uberlândia, Uberlândia-MG, 2017.

\section{Endereço para Correspondência}

Universidade Federal de Goiania - UFG

Av. Esperança, $s / n$ - Chácaras de Recreio Samambaia, Goiânia - GO

CEP.: 74690-900

e-mail: flavia.fernandes92@gmail.com

Recebido em 18/09/2018

Aprovado em 01/04/2019

Publicado em 13/08/2019 